\title{
The relationships between the immunoexpression of KAI1, MMP-2, MMP-9 and steroid receptors expression in endometrial cancer
}

\author{
Anna Grybos ${ }^{1}$, Julia Bar ${ }^{2}$ \\ ${ }^{1}$ Department of Gynecology and Obstetrics, Wroclaw Medical University, Wroclaw, Poland \\ ${ }^{2}$ Department of Pathomorphology and Oncological Cytology, Wroclaw Medical University, \\ Wroclaw, Poland
}

\begin{abstract}
The role of the parallel expression of the KAI1 protein and metalloproteinases (MMP-2 and MMP-9) in respect to the status of steroid receptors in endometrial cancer is still incompletely understood. The aim of this study was to evaluate the expression of and correlation between KAI1 on one hand and MMP-2 and MMP-9 on the other hand in terms of the status of the estrogen (ER) and progesterone receptors (PR) in 100 patients with endometrial cancer. The expressions of KAI1, MMP-2, MMP-9, ER and PR were assessed immunohistochemically on paraffin-embedded tissues. No correlations were found between these biomarkers and the clinical and pathological parameters of the endometrial cancer. However, in KAI1-positive cases, the expression was limited to a small area of tumor tissue in FIGO stages III-IV. A tendency towards the high expression of MMP-9 and MMP-2 was observed in the advanced stages of endometrial cancer (FIGO IIIA-IV). Positive correlations between the presence of KAI1 and PR and between the presence of MMP-9 and PR were found in endometrial cancer. A positive correlation was also observed between KAI1 and MMP-2 expression, and a borderline one between KAI1 and MMP-9 expression in endometrial cancer. $\mathrm{KAI} 1^{+} / \mathrm{PR}^{+}$and $\mathrm{KAI}^{+} / \mathrm{ER}^{-}$ immunophenotypes were observed more frequently in FIGO low stages and with well-differentiated tumor grade. However, the KAI1-/ER ${ }^{+}$and $\mathrm{KAI} 1^{-} / \mathrm{PR}^{+}$immunophenotypes were mainly observed in advanced stages of endometrial cancer. KAI1 $/$ MMP- $^{+}$and $\mathrm{KAI} 1^{+} / \mathrm{MMP}-9^{+}$immunophenotypes were observed in FIGO I stage and with well-differentiated tumors. KAI1 ${ }^{-} / \mathrm{MMP}^{+} 2^{+}$and $\mathrm{KAI} 1^{-} / \mathrm{MMP}-9^{+}$phenotypes were more often observed in FIGO stage II. Our study showed that KAI1 protein, as well as steroid receptors, might modulate MMP-2 and MMP-9 expression in endometrial cancer. Our study revealed that the overlapping expression of the biomarkers investigated here suggests that cooperation between these molecules exists, even at the early stages of endometrial cancer growth, and may determine the speed of tumor cell dissemination and might characterize the biological behavior of endometrial cancer. (Folia Histochemica et Cytobiologica 2014, Vol. 52, No. 3, 187-194)
\end{abstract}

Key words: endometrial cancer; KAI1 protein; MMP-2; MMP-9; steroid receptors; immunohistochemistry

\section{Introduction}

Endometrial cancer is the most common malignancy of the female genital tract in developed countries [1-3]. The mortality rate in Europe is about 9000 per

\footnotetext{
Correspondence address: A. Grybos M.D., Ph.D.

Department of Gynecology and Obstetrics

Wroclaw Medical University

Bartla St. 5, 51-618 Wroclaw, Poland

tel.: +48602790667

e-mail: annagrybos@yahoo.pl
}

year. According to Cancer Incidence in Five Continents and the International Agency for Research on Cancer, the global morbidity rate is $6.5 \%$ and the global mortality rate is about $1.6 \%$ [1-4].

The risk factor is based on the range of glandular and nonglandular epithelial components [5]. In endometrial cancer, even in cases diagnosed at stages I and II of the FIGO classification, a cancer recurrence or metastatic spread can appear [6]. To assess the risk of recurrence in patients with endometrial cancer, many biological factors, such as tyrosine kinase receptors, steroid receptors, and proteins associated with me- 
tastasis, have been analyzed [7]. For a solid tumor, the neoplastic cell population must spread from the primary tumor to the surrounding tissues, in order to find an area amenable to growth. This process may occur at an early stage of tumor development [8]. Park et al. [9] revealed that, during metastases, different signaling pathways in tumor cells may be activated. An experimental study revealed that this expansion can be clonal and limited to a more biological aggressive subpopulation of tumor cells [9]. The first stage of tumor invasion involves degradation of the basement membrane, caused selectively by metalloproteinases (MMPs) [10]. MMPs play important roles in invasion and metastasis by regulating the signaling pathways that control cell growth and invasion [10]. Of all the metalloproteinases, the type-IV collagenase group of MMP-2 and MMP-9 has been linked to endometrial cancer progression and invasion [6]. Expression of different factors facilitates a subclone of a tumor cell growing to micrometastasis, allowing tumor cell outgrowth at a new, distant site [10]. Upexpression and downexpression of several genes that play different roles in a cell - including metastasis suppressor genes - has been described as occurring in the metastatic process [11]. KAI1 is a member of the tetraspan transmembrane superfamily (TM4SF) of type-III membrane proteins. It was originally identified as a metastasis suppressor in prostate cancer [12]. KA1 is an important tumor metastasis suppressor gene whose inactivation plays a crucial role in the tumor metastasis process [11, 12]. KAI1 expression is frequently downregulated in many human cancers, including endometrial cancer [13]. Experimental data has shown that the KAI1 protein may mediate growth-suppressive signals, which lead to the induction of the p21 protein in melanoma cells [11]. Additionally, KAI1 interacts with different proteins, such as epidermal growth factor receptor (EGFR) and Src (intracellular tyrosine kinase) and plays a role in a functional signaling network that influences the metastatic potential of cancer [9].

The role of MMP and KAI1 expression in tumor metastasis is still controversial $[12,14,15]$. Moreover, some reports have revealed their important roles in endometrial cancer invasion [15]. Similarly, there is data that MMP expression is regulated by different factors, such as $p 53$, p48, ETS, and ERK1/2 [8, 10]. To our knowledge, there are no studies that analyzed the relationship between the expression of the KAI1 protein, MMP-2, and MMP-9 in endometrial cancer in respect to steroid receptor status, with the aim of showing whether these proteins are jointly involved in the invasive growth of endometrial cancer. The present study evaluates the expression of and the correlation between KAI1 and MMP-2 and MMP-9 in respect to the status of estrogen receptors (ER) and progesterone receptors (PR) in endometrial cancer.

\section{Material and methods}

Patients. From 1995 to 2008, tissue samples were collected from 100 patients diagnosed with endometrial cancer undergoing surgery in the First Clinic of Gynecology, Wroclaw Medical University in Poland. None of the women had received radiotherapy or chemotherapy prior to surgery. The tumor tissues were histologically verified to confirm the diagnosis, histological type, and grade, according to the World Health Organization criteria [16] and the FIGO classification (2009).

Immunohistochemistry. Immunohistochemical staining for the proteins under investigation was performed on paraffin-embedded tissue using the Universal DakoCytomation $\mathrm{LSAB}+$ Kit peroxidase procedure (LSAB + Kit:HRP, Dako, Copenhagen, Denmark). The tissue samples were treated with primary monoclonal mouse antibodies against steroid receptors ER (clone 6F11, Novocastra, Newcastle, UK) and PR (clone 1A6, Novocastra), against the metalloproteinases MMP-2 (clone 17B11, Novocastra) and MMP-9 (clone 203, Novocastra), and against the metastatic suppressor protein KAI1 (clone G-2, Santa Cruz Biotechnology, Santa Cruz, CA, USA). Dilutions of 1:50, 1:50, 1:80, 1:40, and 1:50 of the stock primary antibodies were used for ER, PR, MMP-2, MMP-9, and KAI1 detection, respectively.

Five-micrometer-thick sections from one selected block from each lesion were deparaffinized and boiled for $3 \times 5 \mathrm{mi}-$ nutes for each antibody in a citrate buffer $(\mathrm{pH} 6.0)$ at $700 \mathrm{~W}$ in a microwave oven. After the microwave treatment, the tissue sections were cooled slowly for 20 minutes. Endogenous peroxidase reactivity was blocked with $3 \% \mathrm{H}_{2} \mathrm{O}_{2}$ and nonspecific tissue reactions with $10 \%$ bovine serum albumin. Tissue specimens were incubated with primary monoclonal antibodies. Following washing with $0.1 \mathrm{M}$ Tris-buffer of $\mathrm{pH}=7.4$ (TBS), the tissue specimens were incubated with secondary biotinylated rabbit antibody (Dako), anti-mouse IgG and with streptavidin conjugated to horseradish peroxidase (Dako), both for 15 minutes at room temperature. After washing with TBS, the antigen-antibody reaction was visualized with DAB (3,3-diaminobenzidine) (Dako) as a chromogen ( $8 \mathrm{~min}$, room temperature). Sections were counterstained with hematoxylin and mounted. The incubation buffer (TBS), without a primary antibody, was used as a negative control. The internal positive controls were performed according to manufacturer's procedure to confirm the specificity of the immunostaining. The following positive controls were used: lobular breast carcinoma for estrogen and progesterone receptors, sarcoma tissue for MMP-2, macrophages for MMP-9, and tonsil for KAI1 protein. 
Interpretation of immunostaining results. The preparations were evaluated under a BH-2 Olympus light microscope (Olympus, Tokyo, Japan). The localization, distribution, and intensity of the immunostaining were assessed in the tissue sections. The semiquantitative evaluation of the IHC staining was performed without knowledge of the clinical data, and a consensus was reached on controversial cases. To evaluate the PR and ER, we applied the criteria used in endometrial adenocarcinoma immunostaining [2].

The nuclear immunostaining for estrogen and progesterone receptors was recorded as the percentage of positively stained cells with positive samples, defined as those showing more than $10 \%$ stained cells. For MMP-2 and MMP-9, cytoplasmic immunostaining (and for KAI1 membrane immunostaining) was considered as positive when more than $10 \%$ of the tumor tissue showed immunopositivity $[14,15]$. The intensity of staining was scored 0 for negative, + for weak, ++ for moderate, and +++ for strong.
Statistical analysis. The correlations between the steroid receptors (ER, PR) and MMP-2, MMP-9, and KAI1 protein expression with the clinical and pathological parameters of the endometrial carcinomas were statistically evaluated using the chi-square test. Associations between the biomarkers were analyzed using the Spearman rank correlation. Differences were considered significant at $p \leq 0.05$.

\section{Results}

All the biomarkers investigated showed a heterogeneous pattern of immunoreactivity. Depending of the markers assessed, positive immunostaining was observed in $10 \%$ to $90 \%$ of tumor lesions. Positive nuclear expression of ER and PR was found in $46.0 \%$ and $51.0 \%$ of endometrial cancer cases, respectively. Strong expression of both steroid receptors was found in the glandular structure of the tissue (Figure 1A, B). Diffuse
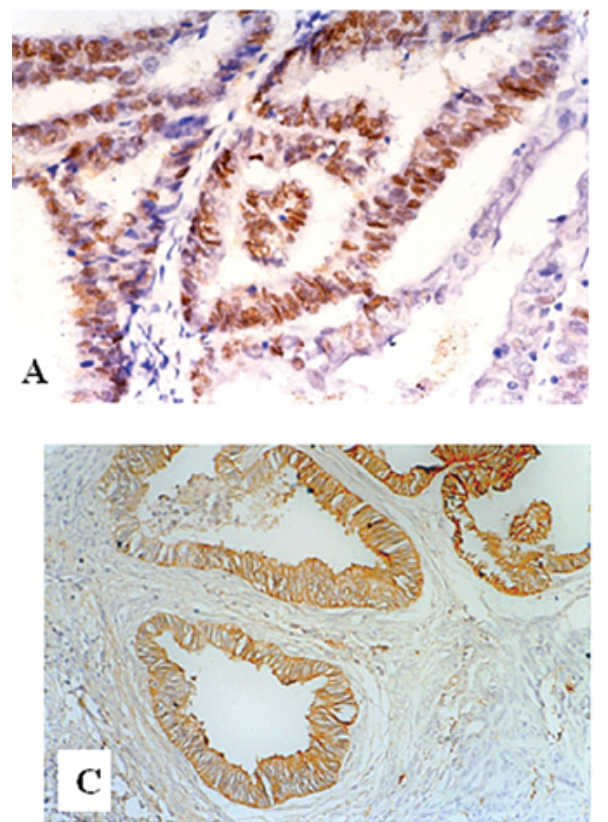
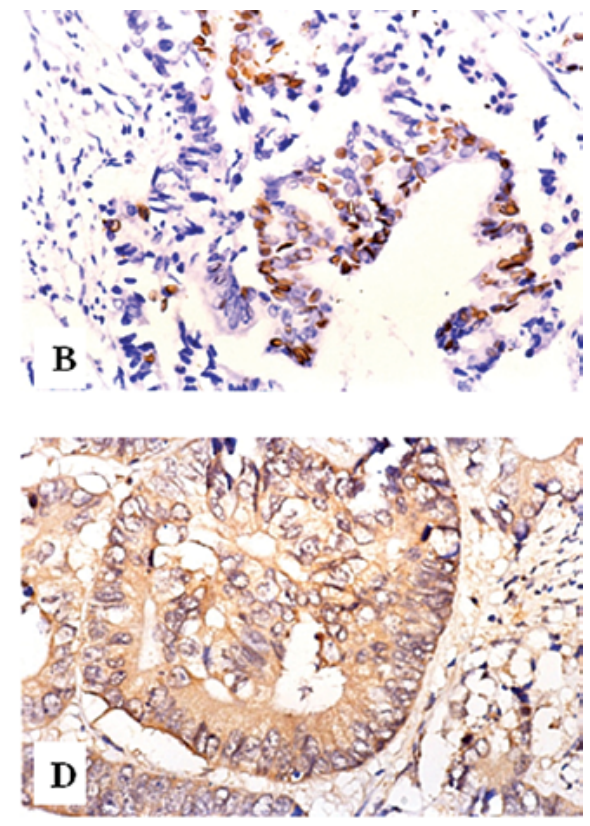

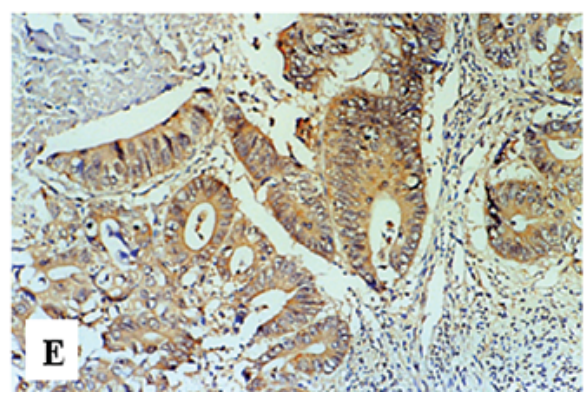

Figure 1. Immunoexpression of the studied proteins in the endometrial cancer. The nuclear expression of (A) estrogen (ER) and (B) progesterone (PR) receptors. The cytoplasmic localization of MMP-2 (C) and MMP-9 (D). The membrane localization of KAI1 (E). Immunostaining was performed using the avidin-biotin staining (ABC method) as described in Methods. Total magnification $\times 400$ 
Table 1. Expression of KAI1, MMP-2, MMP-9, ER, and PR in endometrial cancers in relation to clinical and pathological parameters

\begin{tabular}{|l|c|c|c|c|c|c|c|}
\hline $\begin{array}{l}\text { Clinical and pathological } \\
\text { parameters }\end{array}$ & \multicolumn{7}{|c|}{ Number of immunoreactive cases (percentage) } \\
\hline & $\begin{array}{c}\text { Number } \\
\text { of patients }\end{array}$ & KAI1+ (\%) & ER $^{+}(\%)$ & PR $^{+}(\%)$ & $\begin{array}{c}\text { MMP-2+ } \\
(\%)\end{array}$ & $\begin{array}{c}\text { MMP-9+ }^{+} \\
(\%)\end{array}$ & $p_{\text {-value }}$ \\
\hline FIGO IA & 47 & $28(59.5)$ & $18(38.2)$ & $21(44.6)$ & $19(40)$ & $26(55.3)$ & NS \\
\hline FIGO IB-II & 46 & $27(58.6)$ & $19(41.3)$ & $26(56.2)$ & $18(39.1)$ & $28(60.8)$ & NS \\
\hline FIGO IIIA-IVB & 7 & $5(71.4)$ & $4(57.1)$ & $4(57.1)$ & $4(57.1)$ & $5(71.4)$ & NS \\
\hline TOTAL & $\mathbf{1 0 0}$ & $\mathbf{6 0}(\mathbf{6 0})$ & $\mathbf{4 1 ( 4 1 )}$ & $\mathbf{5 1}(\mathbf{5 1})$ & $\mathbf{4 1 ( 4 1 )}$ & $\mathbf{5 9}(\mathbf{5 9})$ & NS \\
\hline G1 & 67 & $41(61.1)$ & $25(37.3)$ & $33(49.2)$ & $28(41.7)$ & $39(58.2)$ & NS \\
\hline G2 $(\mathrm{n}=24)+\mathbf{G 3}(\mathrm{n}=9)$ & 33 & $19(57.5)$ & $16(48.4)$ & $18(54.3)$ & $13(39.3)$ & $20(60.6)$ & NS \\
\hline TOTAL & $\mathbf{1 0 0}$ & $\mathbf{6 0}(\mathbf{6 0})$ & $\mathbf{4 1}(\mathbf{4 1})$ & $\mathbf{5 1}(\mathbf{5 1})$ & $\mathbf{4 1}(\mathbf{4 1})$ & $\mathbf{5 9}(\mathbf{5 9})$ & NS \\
\hline
\end{tabular}

G1, G2, G3 - grade of tumor differentiation; NS — not significant

cytoplasmic localization of MMP-2 and MMP-9 was observed in $41.0 \%$ and $59.0 \%$ of endometrial cancers, respectively. The expression of MMP-2 and MMP-9 dominated in the center of the tissue and was reduced at the edge of the carcinoma structure (Figure 1C, D). In half of all cases, MMP-2 and MMP-9 expression was found in stromal tissue surrounding the tumor structures; however, the immunostaining was stronger in the tumor cells than in the stromal cells. Membrane KAI1 protein expression was found in $60.0 \%$ of cases. The cells expressing KAI1 protein formed nests, and were distributed in tubular structures of neoplastically altered tissue (Figure 1E). Heterogeneous staining for KAI1 protein was observed not only between different endometrial cancer cases, but also in individual cases. KAI1 expression was not found in stromal tissue.

No correlations were seen between the studied biomarkers and the clinical or pathological parameters of endometrial cancer (see Table 1). Interestingly, in KAI1-positive cases, the expression was dependent on the stage of the disease and was limited to a small area of tumor tissue in FIGO stages III-IV. A tendency towards the high expression of MMP-9 and MMP-2 was found in the advanced stages of endometrial cancer (FIGO IIIA-IV). The differences were not statistically significant $(p>0.05)$. The expression of all the biomarkers was analyzed by tumor grade G1 versus grade $\mathrm{G} 2 / \mathrm{G} 3$. Independent of tumor grade, the expression of all proteins was comparable (Table 1).

In further analysis, we estimated the association between steroid receptor, KAI1, and metalloproteinase (MMP-2, MMP-9) expression in the whole group of endometrial cancer, as well as for the FIGO stage and tumor grade, to determine the overlapping effect of the investigated biomarkers in endometrial cancer progression.
Positive correlations between KAI1 protein and PR, and between MMP-9 and PR presence, were found in endometrial cancer $(p=0.01, \mathrm{r}=+0.24$; $p=0.02, \mathrm{r}=+0.22$ respectively) (Table 2 ). A positive correlation was observed between KAI1 and MMP-2 expression $(p=0.04 ; \mathrm{r}=+0.19)$ and a borderline correlation was seen between KAI1 and MMP-9 $(p=0.07 ; \mathrm{r}=+0.18)$ in endometrial cancers (Table 3$)$.

Taking into account the level of MMP-2, MMP-9, and KAI1 concomitant expression in endometrial cancer samples in relation to ER and PR expression, it is worth underlining that the $\mathrm{KAI} 1^{+} / \mathrm{PR}^{+}$and $\mathrm{KAI}^{+} / \mathrm{ER}^{-}$immunophenotypes were observed more frequently in low FIGO stage and well-differentiated tumor grades. In contrast, $\mathrm{KAI} 1^{-} / \mathrm{ER}^{+}$and $\mathrm{KAI} 1^{-} / \mathrm{PR}^{+}$ immunophenotypes were mainly observed in the advanced stages of endometrial cancer. Endometrial cancers showing $\mathrm{KAI}^{+} / \mathrm{MMP}-2^{+}$or $\mathrm{MMP}^{-} 9^{+}$ immunophenotypes were observed in FIGO stage I and well-differentiated tumors. KAI1-/MMP-2 ${ }^{+}$and MMP $-9^{+}$cases, however, were observed in FIGO stage II (Table 4).

\section{Discussion}

The key step in endometrial cancer invasion involves the degradation of extracellular matrix components and the migration of cancer cells into other organs [10]. Invasion by tumor cells is characterized by a down-regulation or upregulation of metastasis suppressor genes [12]. KAI1 is a basic protein that determines the motility and invasiveness of cells [14]. So far, the biology and the interaction of KAI1 with other proteins related to metastasis are unknown. In line with previous result $[13,15,17,18]$, we found in this study that the expression of steroid receptors, metallopro- 
Table 2. Correlation between KAI1, MMP-2, and MMP-9 expression and steroid receptors expression in endometrial cancers

\begin{tabular}{|c|c|c|c|c|c|c|}
\hline \multicolumn{7}{|c|}{ Immunoreactivity } \\
\hline & \multicolumn{3}{|c|}{$\begin{array}{c}\text { Progesterone receptor (PR) } \\
\text { (number of positive or negative cases) }\end{array}$} & \multicolumn{3}{|c|}{$\begin{array}{c}\text { Estrogen receptor }(\mathrm{ER}) \\
\text { (number of positive or negative cases) }\end{array}$} \\
\hline & $\mathbf{P R}^{+}$ & $\mathbf{P R}^{-}$ & & $\mathbf{E R}^{+}$ & $\mathbf{E R}^{-}$ & \\
\hline KAI1 $^{+}$ & 35 & 25 & \multirow{2}{*}{$\begin{array}{c}p=0.01 \\
R=+0.24\end{array}$} & 23 & 37 & \multirow{2}{*}{ NS } \\
\hline KAI1- & 16 & 24 & & 18 & 22 & \\
\hline MMP-2+ & 20 & 21 & \multirow{2}{*}{ NS } & 16 & 25 & \multirow{2}{*}{ NS } \\
\hline MMP-2- & 30 & 29 & & 25 & 34 & \\
\hline MMP-9+ & 33 & 26 & \multirow{2}{*}{$\begin{array}{c}p=0.02 \\
\mathrm{R}=+\mathbf{0 . 2 2}\end{array}$} & 24 & 35 & \multirow{2}{*}{ NS } \\
\hline MMP-9- & 18 & 23 & & 17 & 24 & \\
\hline
\end{tabular}

$\mathrm{R}$ - rank correlation; $p-p$-value; NS — not significant

Table 3. Correlations between examined biomarkers in endometrial cancers

\begin{tabular}{|c|c|c|c|}
\hline \multicolumn{4}{|c|}{ Immunoreactivity (number of positive or negative cases) } \\
\hline Proteins studied & KAI1 $^{+}$ & KAI1- $^{-}$ & \\
\hline MMP-2+ & 30 & 11 & \multirow{2}{*}{$\begin{array}{c}p=0.04 \\
R=+0.19\end{array}$} \\
\hline MMP-2- & 30 & 29 & \\
\hline MMP-9+ & 39 & 20 & \multirow{2}{*}{$\begin{array}{c}p=\mathbf{0 . 0 7} \\
R=+\mathbf{0 . 1 8}\end{array}$} \\
\hline MMP-9' & 21 & 20 & \\
\hline
\end{tabular}

$\mathrm{R}$ - rank correlation; $p-p$-value

teinases, and KAI1 protein was observed in similar percentages of endometrial cancers.

In contrast to the current study, there is in the literature data that shows the association between MMP-2 and MMP-9 expression and the FIGO stage of endometrial cancer [19]. However, there is also data that, like our results, reveals no correlation between the examined MMPs and clinical and pathological parameters $[6,7,15]$. It is worth mentioning that, in our study, the expression of MMP-2 and MMP-9 was observed mainly in cases with advanced FIGO stage, and that MMP-9 immunopositivity was found in a greater area of tissue than in the case of cancers with less advanced FIGO stages.

The increased expression of MMP-2 and MMP-9 in advanced stages of endometrial cancer indicates that both enzymes are involved in tumor progression and may play an important role in the dissemination of tumor cells acting via basement membrane degradation $[10,15]$. On the other hand, recent data shows that MMPs play mainly an indirect role in the cell signaling pathway through the activity of biomolecules that target specific receptors that are responsible for cell growth and migration. Such a mechanism can be considered during endometrial cancer growth as a hormone-dependent tumor [19].

In agreement with earlier data on KAI1 expression in gastric, breast, head and neck cancers [14, 20, 21], we observed a loss of KAI1 protein expression in almost half of primary endometrial cancers. KAI1-negative tumors were found frequently in poorly differentiated cases, similarly to the situation previously described with gastric cancer [20]. In our study, KAI1 downregulation was not significantly associated with an advanced clinical stage of disease. This may be due to the limited number of cases in FIGO III and IV. Nevertheless, we observed the trend toward KAI1 downregulation in the form of increased stages of endometrial tumor, consistent with the postulated role of KAI1 as a suppressor protein of tumor aggressive growth [11].

Increasing attention has been paid to the interaction between different factors involved in metastatic processes in hormone-sensitive tumors [22-24]. To our knowledge, this is the first study which has analyzed the association between steroid receptors, KAI1 protein, MMP-2, and MMP-9 - as well as between KAI1, MMP-2, and MMP-9 
Table 4. Distribution of different immunophenotypes in relation to the clinicopathological parameters of endometrial cancer

\begin{tabular}{|c|c|c|c|c|}
\hline \multirow[t]{2}{*}{ Clinical and pathological parameters } & \multicolumn{4}{|c|}{$\begin{array}{l}\text { Immunophenotypes of endometrial cancer } \\
\text { (number of cases, all cases } n=100 \text { ) }\end{array}$} \\
\hline & $\mathbf{K A I I}^{+} / \mathbf{E R}^{+}$ & $\mathbf{K A I 1}^{+} / \mathbf{E R}^{-}$ & $\mathbf{K A I 1}^{+} / \mathbf{P R}^{+}$ & $\mathbf{K A I 1}^{+} / \mathbf{P R}^{-}$ \\
\hline FIGO IA & 10 & 18 & 17 & 11 \\
\hline FIGO IB-II & 9 & 17 & 14 & 13 \\
\hline G1 & 12 & 29 & 23 & 20 \\
\hline G2 & 7 & 7 & 7 & 4 \\
\hline \multirow[t]{2}{*}{ G3 } & 4 & 1 & 5 & 1 \\
\hline & $\mathbf{K A I 1}^{-} / \mathbf{E R}^{+}$ & $\mathbf{K A I 1}^{-} / \mathbf{E R}^{-}$ & $\mathbf{K A I 1}^{-} / \mathbf{P R}^{+}$ & $\mathbf{K A I 1}^{-} / \mathbf{P R}^{-}$ \\
\hline FIGO IA & 8 & 11 & 4 & 15 \\
\hline FIGO IB-II & 10 & 9 & 12 & 7 \\
\hline G1 & 12 & 15 & 10 & 16 \\
\hline G2 & 6 & 5 & 5 & 5 \\
\hline \multirow[t]{2}{*}{ G3 } & 2 & 2 & 1 & 3 \\
\hline & KAI1 $^{+} /$MMP-9 $^{+}$ & KAI1 $^{+} /$MMP-9- $^{-}$ & KAI1 $^{+} / \mathrm{MMP}^{-2}{ }^{+}$ & $\mathrm{KAI1}^{+} / \mathrm{MMP}^{-2}$ \\
\hline FIGO IA & 19 & 9 & 15 & 13 \\
\hline FIGO IB-II & 17 & 10 & 11 & 16 \\
\hline G1 & 28 & 29 & 23 & 20 \\
\hline G2 & 8 & 7 & 7 & 4 \\
\hline \multirow[t]{2}{*}{ G3 } & 4 & 1 & 5 & 1 \\
\hline & KAI1-/MMP-9 $^{+}$ & KAI1-/MMP-9- & KAI1-/MMP-2+ & KAI1-/MMP-2- \\
\hline FIGO IA & 7 & 12 & 4 & 15 \\
\hline FIGO IB-II & 11 & 9 & 7 & 12 \\
\hline G1 & 12 & 14 & 8 & 18 \\
\hline G2 & 4 & 5 & 2 & 8 \\
\hline G3 & 4 & 0 & 1 & 3 \\
\hline
\end{tabular}

G1, G2, G3 - grades of tumor differentiation

- in endometrial cancer. Earlier studies revealed that KAI1 protein may cooperate with MMPs, and displays a different role in its functions in ER-negative and ER-positive breast cancers [14, 22, 23]. Christgen et al. [22, 23] found that in ER-positive breast cancers the KAI1 protein was downregulated. However, more aggressive breast cancers commonly express KAI1 protein. In our study, KAI1 expression was independent of ER status in endometrial cancer but was dependent on PR expression. The absence of correlation between KAI1 protein and ER expression in the present study contradicts the data obtained by Christgen et al., who reported the downregulation of KAI1 expression by estrogen [22]. There is no data describing any association between KAI1 and PR expression in human tumors. In our study, PR-positive endometrial cancers overexpressed the KAI1 protein. These results indicate that the mechanism which acti- vates the biological function of KAI1 in endometrial cancers is different than in the case of breast cancer [23]. The positive correlation between KAI1 protein expression and PR in endometrial cancer reveals the cooperation between those proteins, and the synergic inhibiting effect of both proteins could be considered in these cases. These suggestions might be supported by the presence of parallel KAI1/PR expression in early stages of endometrial cancer. On the other hand, the data indirectly suggests that PR expression, as with other receptors, may enhance KAI1 expression, thus suppressing the mobility of the tumor cells and their migration from the tumor mass to surrounding tissues [21]. The differences between our study and other studies might be explained by the different source of biological material. In contrast to our study on tumor tissue, most results describing the metastatic ability of tumor cells have been performed 
on cell lines or animal models $[8,25,26]$. The results from in vitro studies are not comparable with the data from tumor tissue, which is not as homogenic as a cell line $[25,26]$. The results of the present study show that MMP-9 expression was significantly higher in patients with a high level of PR, and that there was a significant positive correlation between MMP-9 and PR, but not between MMP-9 and ER expression, as well as between MMP-2, ER, and PR in endometrial cancer. These results differ from those reported for breast tumor tissue sections [27, 28], which found that ER-negative breast cancer had higher expression of MMP-9, while PR-positive breast cancer had high levels of MMP-2 expression.

There is a lack of published reports regarding possible associations between the status of steroid receptors and the expression of MMP-2 and MMP-9 in endometrial cancer. However, some results imply that MMP-2 and MMP-9 expression could be hormonally regulated in endometrial cancer. This suggestion might be supported by data which shows that the expression of MMP-2 depends on PR isoforms. PR-B has been found to decrease MMP-2 expression and PR-A to increase MMP-2 expression in human deciduas [29]. In our study, we might consider such regulation of MMP- 2 and MMP-9 by isoforms of $\mathrm{ER} \alpha, \beta$, PR-A, and PR-B playing different biological functions in tumor cells [19]. Our data, showing a positive relation between KAI1 and MMP-2 expression in endometrial cancers, are partly consistent with recently published results which have shown a relationship between the expression of KAI1, MMP-2, and MMP-9 in bladder cancer cell lines [30]. The authors of that study showed that poorly invasive KAI1-positive cell lines express high levels of MMP-2 mRNA and (pro-MMP-2) protein, whereas cell lines that are KAI1-negative and possess an invasive phenotype show no detectable MMP-2 mRNA expression [30]. The same study indicated that some cell lines showed pro-MMP-2 protein but no gelatinolytic activity, and its authors suggested that the levels of KAI1 might be directly related to MMP-2 mRNA and MMP-2 activity [30]. In our present study, we analyzed the expression of the KAI1 and MMP-2 proteins, and not the mRNA level of the MMP-2 gene. Our results, however, showed the association between both proteins existing in endometrial cancer in early FIGO stage, which could suggest that the KAI1 protein might reduce the gelatinolytic activity of MMP-2 [11, 30]. Similarly to the results of recent reports, no association was found between KAI1 and MMP-9 expression in endometrial cancer [30]. Based on analyses comparing immunophenotypes with the clinicopathological parameters of endometrial cancers, we can suggest that, of the studied proteins, KAI1 expression is the most important factor in the progression of the tumor in the early stages of endometrial cancer growth.

In conclusion, our results show that KAI1 protein, as well as steroid receptors, might modulate the level of MMP-2 and MMP-9 expression in endometrial cancer. The positive and negative cooperation between these biomarkers exists in the early stages of endometrial cancer growth, and might determine the speed of the dissemination of tumor cells and reflect the biological behavior of the tumor.

\section{Acknowledgments}

We are grateful to Professor Jerzy Rabczynski for the histological verification and to Professor Marian Grybos for the endometrial cancer tissue samples.

\section{References}

1. Amant F, Moerman P, Neven P. Endometrial cancer. Lancet 2005;6-12:491-505.

2. Nicholson MR, Iyengar P, Hummer AJ, Linkov I, Asher M, Soslow RA. Immunophenotypic diversity of endometrial adenocarcinomas: implications for differential diagnosis. Modern Pathol. 2009;19:1091-1100.

3. Shabani N, Kuhn C, Kunze S et al. Prognostic significance of oestrogen receptor alpha (ERalpha) and beta (ERbeta), progesterone receptor A (PR-A) and B (PR-B) in endometrial carcinomas. Eur J Cancer. 2007;43:2434-2444.

4. Hidaka T, Kato K, Yonezawa $\mathrm{R}$ et al. Omission of lymphadenectomy is possible for low-risk corpus cancer. Eur J Surg Oncol. 2007;3:86-90.

5. Pilka R, Norata GD, Domański H et al. Matrix metalloproteinase-26 (Matrilysin-2) expression is high in endometrial hyperplasia and decreases with loss of histological differentiation in endometrial cancer. Gynecol Oncol. 2004;94:661-670.

6. Honkavuori M, Talvensaari-Mattila A, Soini Y et al. MMP-2 expression associates with CA 125 and clinical course in endometrial carcinoma. Gynecol Oncol. 2007;104:217-221.

7. Steinbakk A, Skaland I, Gudlaugsson E et al. The prognostic value of molecular biomarkers in tissue removed by curettage from FIGO stage 1 and 2 endometrioid type endometrial cancer. Am J Obstet Gynecol. 2009;200:78.e8.

8. Geiger TR, Peeper DS. Metastasis mechanisms. Bioch Bioph Acta. 2009;1796:293-308.

9. Park JJ, Jin YB, Lee YJ, Lee YS, Ko YG, Lee M. KAI 1 suppresses HIF-1 $\alpha$ and VEGF expression by blocking CDDP-1 enhanced Src activation in prostate cancer. BMC Cancer. 2012;12:81.

10. Bauvois B. New facets of matrix metalloproteinases MMP-2 and MMP-9 as cell transducers: outside-in signaling and relationship to tumor progression. Biochim Biophys Acta. 2012;1825:29-36.

11. Tang Y, Cheng Y, Martinka M, Ong CJ, Li G. Prognostic significance of KAI1/CD82 in human melanoma and its role in cell migration and invasion through the regulation of ING4. Carcinogenesis. 2014;35:86-95.

12. Stafford LJ, Vaidya KS, Welch DR. Metastasis suppressors genes in cancer. Int J Biochem Cell Biol. 2008;40:874-891.

13. 13. Liu FS, Dong JT, Chen JT et al. KAI1 metastasis suppressor protein is down-regulated during the progression of human endometrial cancer. Clin Cancer Res. 2003;9:1393-1398. 
14. Zhang T, Ren G, Zhang Z, Zhang R, Li Y. The expression of tumor metastasis suppressor gene KAI1 and matrix metalloproteinase 2 in breast cancer tissue. Afr J Pharm Pharmacol. 2012;6:1923-1926.

15. Weigel MT, Kramer J, Schem C et al. Differential expression of MMP-2, MMP-9 and PCNA in endometriosis and endometrial carcinoma. Eur J Obstet Gynecol Rep Biol. 2011;160:74-78.

16. Creasman W. Revised FIGO staging for carcinoma of the endometrium. Int J Gynecol Obstet. 2009;105:109.

17. Aglund K, Rauvala M, Puistola U et al. Gelatinases A and B (MMP-2 and MMP-9) in endometrial cancer-MMP-9 correlates to the grade and the stage. Gynecol Oncol. 2004;94:699-704.

18. Briese J, Schutle HM, Sajin M et al. Correlations between reduced expression of the metastasis suppressor gene KAI 1 and accumulation of 553 in uterine carcinomas and sarcomas. Virchows Arch. 2008;453:489.

19. Bender D, Buekers T, Leslie KK. Hormones and receptors in endometrial cancer. Proc Obstet Gynecol. 2011;2:1-25.

20. Knoener M, Krech T, Puls F, Lehmann U, Kreipe H, Christgen M. Limited value of KAI1/CD82 protein expression as a prognostic marker in human gastric cancer. Disease Markers. 2012;32:337-342.

21. Zhang B-H, Liu W, Li L et al. KAI1/CD82 and MRP1/CD9 serve as markers of infiltration, metastasis, and prognosis in laryngeal squamous cell carcinomas. Asian Pacific J Cancer Prev. 2013;14:3521-3526.

22. Christgen M, Christgen H, Heil C et al. Expression of KAI1/ /CD82 in distant metastases from estrogen receptor-negative breast cancer. Cancer Sci. 2009;100:1767-1771.
23. Christgen M, Bruchhardt H, Ballmaier M et al. KAI1/CD82 is a novel target of estrogen receptor-mediated gene repression and downregulated in primary human breast cancer. Int J Cancer. 2008;123:2239-2246.

24. Lee HL, Park I, Byuan HJ, Jeoung D, Kim YM, Lee H. Metastasis suppressor KAI1/CD82 attenuates the matrix adhesion of human prostate cancer cells by suppressing fibronectin expression and $\beta_{1}$ integrin activation. Cell Physiol Bichem. 2011;27:575-587.

25. Wan L, Pantel K, Kang Y. Tumor metastasis: moving new biological insights into the clinic. Nature Med. 2013;19:1450-1464 .

26. Ribells N, Santoja A, Pajares B, Liacer C, Alba E. The seed and soil hypothesis revisited: current state of knowledge of inherited genes on prognosis in breast cancer. Cancer Treat Rev. 2014;40:293-299.

27. Daniel A, Zito AF, Giannelli G et al. Expression of metalloproteinases MMP-2 and MMP-9 in sentinel lymph node and serum of patients with metastatic and non-metastatic breast cancer. Anticancer Res. 2010;30:3521-3528.

28. Milovanovic J, Todorovic-Rakovic N, Rabi ZA. The prognostic role of interleukin-8 (IL-8) and matrix metalloproteinases-2 and -9 in lymph node-negative untreated breast cancer patients. JBUON. 2013;18:867-875.

29. Goldman S, Shalev E. Progesterone receptor isoforms profile, modulate matrix metalloproteinase 2 expression in the deciduas. Am J Obstet Gynecol. 2007;197:e1-e8.

30. You J, Madigan MC, Rowe A, Sajjinovic M, Russell PJ, Jackson P. An inverse relationship between KAI 1 expression and activity in bladder cancer cell lines. Urol Oncol Sem Oncol Invest. 2012;30:502-508. 